\title{
About the automatic generation of equations of curvilinear systems
}

\author{
Jean Lerbet
}

\begin{abstract}
Following other papers devoted to intrinsic formulations of curvilinear systems, we develop here the Maple procedures and some additional calculations in Lie group of displacements which yield explicit scalar equations.
\end{abstract}

Keywords Lie group · Curvilinear system . Symbolic calculation

\section{Introduction}

Using Lie group theory, we have already given the intrinsic equations of any curvilinear system $(\Sigma)$, the only hypothesis being that $(\Sigma)$ is a continuous distribution of rigid bodies called sections (see [3, 4]). The last step for obtaining scalar equations lies in the use of procedures of symbolic calculations allowing the deduction of scalar equations after the parametrization of displacements. The goal of this paper is to highlight the necessary elements to do so. The outline of this paper is as follows: a brief recall of mathematical tools concerning Lie groups is presented in Sect. 2. In Sect. 3 the model and the intrinsic equations are recalled. Section 4 deals with the automatic obtainment

J. Lerbet ( $\square)$

IBISC, FRE CNRS 2873, Université d'Evry Val d'Essone,

40 Rue Pelvoux, CE 1455 Courcouronnes,

91020 Evry Cedex, France

e-mail: jean.lerbet@ibisc.univ-evry.fr of equivalent scalar equations. In the last part, the corresponding Maple procedures and explicit scalar equations are given. Their complexity proves a posteriori the validity of this approach.

\section{Mathematical tools}

Let $\mathcal{E}$ be the affine space of three dimensions of ordinary Euclidean geometry and $E$ the associated vector space. To each affine map $A: \mathcal{E} \rightarrow \mathcal{E}$ is usually associated its linear part $\mathbf{A}$ such that:

$\forall m, p \in \mathcal{E} \quad A(m)=A(p)+\mathbf{A}(\overrightarrow{p m})$.

Let $\mathbb{D}$ be the group of affine mappings $A$ such that $\mathbf{A}$ is an element of the special orthogonal group $S O(E)$. In other words, $\mathbb{D}$ is the group of Euclidean displacements.

Let $\mathcal{D}$ also be the six-dimensional vector space of skew symmetric vector fields $X: \mathcal{E} \rightarrow E$ such that there exists $\omega_{X}$ in $E$ with the well-known following property:

$\forall a, b \in \mathcal{E} \quad X(a)=X(b)+\omega_{X} \wedge \overrightarrow{b a}$.

In other words, the linear part $\mathbf{X}$ of $X$ is the following linear operator in $E$ :

$u \mapsto \mathbf{X}(u)=\omega_{X} \wedge u$

and $\mathcal{D}$ shall be identified with the set of screws. The Lie bracket is defined in $\mathcal{D}$ by $[X, Y](a)=\omega_{X} \wedge$ 
$Y(a)-\omega_{Y} \wedge X(a)$ for any $a$ in $\mathcal{E}$. Thus $\mathcal{D}$ is a Lie algebra which is isomorphic to (and identified with) the classical Lie algebra of $\mathbb{D}$.

The exponential mapping exp $: \mathcal{D} \rightarrow \mathbb{D}$ lets a finite displacement be expressed by its infinitesimal generator and the adjoint mapping $A d: \mathbb{D} \rightarrow \mathcal{L}(\mathcal{D})$ describes how a displacement acts on the elements of $\mathcal{D}$. Thanks to duality, each action on a rigid body shall be described by an element of $\mathcal{D}$ and the operator allowing this identification is the classical inner product $[\cdot \mid \cdot]$ (Klein form in the Lie group theory) defined by:

$[X \mid Y]=\left(\omega_{X} \mid Y(p)\right)+\left(\omega_{Y} \mid X(p)\right)$

the right hand-side being independent of the point $p$ in $\mathcal{E}$. For a more precise discussion about the operations defined on $\mathbb{D}$ and on $\mathcal{D}$, see [1].

\section{The model}

\subsection{Kinematics}

The system is described as follows. The reference configuration is similar to a distribution $\sigma \mapsto r(\sigma)=$ $\left(A(\sigma) ; \vec{i}_{1}(\sigma), \vec{j}_{1}(\sigma), \vec{k}_{1}(\sigma)\right)$ of affine frames where $\sigma$ is the curvilinear abscissa of the curve $\sigma \mapsto A(\sigma)$. $A(\sigma)$ is for example the inertia center of the section of abscissa $\sigma$ and $\vec{i}_{1}(\sigma), \vec{j}_{1}(\sigma), \vec{k}_{1}(\sigma)$ is a basis connected to the rigid section of abscissa $\sigma$ (one may choose for example the Frenet frame of the curve $\sigma \mapsto A(\sigma)$ ). In the following, each rigid section and its associated frame shall be identified.

On each section $r(\sigma)$ and at each time $t$, an unknown displacement $D(\sigma, t)$ acts such that $r(\sigma) \rightarrow$ $r_{a}(\sigma, t)=D(\sigma, t) \bullet r(\sigma)$ where $r_{a}(\sigma, t)=(a(\sigma, t)$; $\left.\vec{i}_{2}(\sigma, t), \vec{j}_{2}(\sigma, t), \vec{k}_{2}(\sigma, t)\right)$. Notice that no hypothesis of perpendicularity of the section with the curve $(\sigma, t) \mapsto a(\sigma, t)$ is made and $\bullet$ is the natural action of $\mathbb{D}$ on the set of affine frames (in mathematical terms it is a structure of fiber bundle).

The kinematics of the system is given by:

- The velocity, described by the following field $v^{c}$ : $[0, l] \times \mathbb{R}^{+} \rightarrow \mathcal{D}:$

$$
(\sigma, t) \mapsto v^{c}(\sigma, t)=\mathbf{D}(\sigma, t)^{-1} \circ \frac{\partial D(\sigma, t)}{\partial t} .
$$

- The acceleration, described by the following field $\dot{v}^{c}:[0, l] \times \mathbb{R}^{+} \rightarrow \mathcal{D}:$

$$
(\sigma, t) \mapsto \dot{v}^{c}(\sigma, t)=\frac{\partial v^{c}(\sigma, t)}{\partial t} .
$$

- The strain, described by the following field $e^{c}$ : $[0, l] \times \mathbb{R}^{+} \rightarrow \mathcal{D}$ :

$$
(\sigma, t) \mapsto e^{c}(\sigma, t)=\mathbf{D}(\sigma, t)^{-1} \circ \frac{\partial D(\sigma, t)}{\partial \sigma} .
$$

One may notice that the field $\sigma \mapsto e^{c}(\sigma, t)$ is unchanged by superposition of a rigid motion.

Remark $1 \frac{\partial D(\sigma, t)}{\partial t}$ and $\frac{\partial D(\sigma, t)}{\partial \sigma}$ belong to $T_{D(\sigma, t)} \mathbb{D}$. So $v^{c}(\sigma, t)$ and $e^{c}(\sigma, t)$ belong $T_{I d} \mathbb{D}$ that is identified with $\mathcal{D}$ as previously remarked.

\subsection{Kinetics and dynamics}

According to the chosen model, we suppose that (at each time $t$ and on each section $\left.r_{a}(\sigma, t)\right)$ there are:

- A distribution $(\sigma, t) \mapsto \mathcal{T}(\sigma, t)$ of moment fields describing the external actions.

- A distribution $(\sigma, t) \mapsto \Theta(\sigma, t)$ of moment fields describing the internal actions, i.e., the action of the right-hand side on the left one (according to the abscissa $\sigma$ ).

- Two concentrated strengths at the ends $\mathcal{T}_{0}(t), \mathcal{T}_{l}(t)$ (in some cases, one could consider a family $\mathcal{T}_{k}(t)$ at $\sigma_{k}$ for $k=1, \ldots, n$ which would introduce a discontinuity of $\sigma \mapsto \Theta(\sigma, t)$ and we should use what follows on each $\left.\left[\sigma_{k}, \sigma_{k+1}\right]\right)$.

- A distribution $\sigma \mapsto H_{r_{a}}(\sigma, t)$ of operators of $\mathcal{D}$ describing the inertial force and torque. As a Lagrangian formulation is used, we need the distribution $\sigma \mapsto H_{r}(\sigma)$ of linear operators of inertia in the reference configuration. The following relation between $H_{r}$ and $H_{r_{a}}$ holds:

$A d(D(\sigma, t)) \circ H_{r}(\sigma) \circ A d^{-1}(D(\sigma, t))=H_{r_{a}}(\sigma, t)$

(for a more precise connection between the operators of inertia $H_{r}$ and $H_{r_{a}}$ and the inertial strength according to the Lagrangian and Eulerian points of view, see [2], for example).

- A distribution $\sigma \mapsto \rho_{0}=\rho_{0}(\sigma)$ of mass density in the reference configuration. 


\subsection{The general equations}

Proposition 1 The equations of the system $(\Sigma)$ are expressed in terms of the Lagrangian (the functions of $\sigma$ and of $t$ are omitted):

$\mathcal{T}^{c}=\rho_{0} H_{r}\left(\dot{v}^{c}\right)+\left[v^{c}, \rho_{0} H_{r}\left(v^{c}\right)\right]-\left[e^{c}, \Theta^{c}\right]-\frac{\partial \Theta^{c}}{\partial \sigma}$,

$\mathcal{T}_{0}^{c}=\Theta^{c}(0)$,

$\mathcal{T}_{l}^{c}=-\Theta^{c}(l)$

where for each object $U=U(\sigma, t)$ attached to $(\Sigma)$, $U^{c}=\operatorname{Ad}\left(D(\sigma, t)^{-1}\right) U$ is the Lagrangian expression of $U$. (See [3] or [4].)

Remark 2 It is clear that this equation, derived from the balance of momentum, is not sufficient for a complete solution of practical problems. A constitutive law must be added to set up a complete system of equations. It is not the subject of the present paper, but we may suppose that, according to the present formulation, such laws in the framework of elasticity or viscoelasticity, for example, should add relations as:

$\Theta^{c}=\mathcal{F}\left(e^{c}, \dot{e}^{c}\right)$.

A future paper shall be devoted to this problem.

\section{Automatic derivation of scalar equations: some elements}

There are several possible representations of a displacement. In order to obtain the scalar equations and to solve them numerically we have to choose one of these representations. Similar remarks may be done for the reference configuration $r(\sigma)$ of the system. Let us now go over the main elements of this program.

1 . There are two parameters noted generically by $w$ : curvilinear abscissa $\sigma$ time $t$.

2. Three bases of $\mathcal{D}$ have been introduced remembering that to each frame $\mathcal{R}=(A ; \vec{i}, \vec{j}, \vec{k})$ of affine space $\mathcal{E}$ is associated a basis $\mathcal{B}=(\mathbf{i}, \mathbf{j}, \mathbf{k}, \xi, \eta, \zeta)$ of $\mathcal{D}$ as follows. To do so, for any $m$ in $\mathcal{E}$, we put:

$$
\begin{aligned}
& \mathbf{i}(m)=\vec{i}, \quad \mathbf{j}(m)=\vec{j}, \quad \mathbf{k}(m)=\vec{k}, \\
& \xi(m)=\vec{i} \wedge \overrightarrow{A m}, \quad \eta(m)=\vec{j} \wedge \overrightarrow{A m}, \\
& \zeta(m)=\vec{k} \wedge \overrightarrow{A m} .
\end{aligned}
$$

The three bases $\mathcal{B}_{0}, \mathcal{B}_{1}, \mathcal{B}_{2}$ are associated to the three fields of affine frames:
- $\mathcal{R}_{0}=\left(A_{0} ; \vec{i}_{0}, \vec{j}_{0}, \vec{k}_{0}\right)$ fixed frame.

- $\mathcal{R}_{1}=\mathcal{R}_{1}(\sigma)=\left(A_{1} ; \vec{i}_{1}, \vec{j}_{1}, \vec{k}_{1}\right)=\left(D_{r}(\sigma)\left(A_{0}\right)\right.$; $\left.\mathbf{D}_{r}(\sigma)\left(\vec{i}_{0}\right), \mathbf{D}_{r}(\sigma)\left(\vec{j}_{0}\right), \mathbf{D}_{r}(\sigma)\left(\vec{k}_{0}\right)\right)$ defines the configuration of reference $r(\sigma)$.

- $\mathcal{R}_{2}=\mathcal{R}_{2}(\sigma, t)=\left(D(\sigma, t)\left(A_{1}\right) ; \quad \mathbf{D}(\sigma, t)\left(\vec{i}_{1}\right)\right.$, $\left.\mathbf{D}(\sigma, t)\left(\vec{j}_{1}\right), \mathbf{D}(\sigma, t)\left(\vec{k}_{1}\right)\right)$ defines the actual configuration at time $t$.

3. The chosen basis or projection basis for obtaining the scalar equations is $\mathcal{B}_{1}$. It is a function of $\sigma$ but we omit to explicit the dependency. Moreover, to represent the element $D(\sigma, t)$, we denote $\left(\rho_{1}^{i}\right)_{i=1,2,3}$ three vectors chosen from the family $\left(\xi_{1}, \eta_{1}, \zeta_{1}\right)$ and write:

$$
\begin{aligned}
D(\sigma, t)= & \exp (u(\sigma, t)) \circ \exp \left(\psi_{1} \rho_{1}^{1}\right) \circ \exp \left(\psi_{2} \rho_{1}^{2}\right) \\
& \circ \exp \left(\psi_{3} \rho_{1}^{3}\right)
\end{aligned}
$$

where $u(\sigma, t)=x \mathbf{i}_{1}+y \mathbf{j}_{1}+z \mathbf{k}_{1}$ is a constant field such that $\exp (u(\sigma, t))$ represents the part of translational motion of $D(\sigma, t)$ and $\exp \left(\psi_{i} \rho_{1}^{i}\right)$ describes the rotation of angle $\psi_{i}$ with respect to the axis of $\rho_{1}^{i}$ for $i=1,2,3$. The choice of the family $\left(\rho_{1}^{i}\right)_{i=1,2,3}$ is free but must represent a whole rotation. The scalar unknowns of the problem are $\left(x, y, z, \psi_{1}, \psi_{2}, \psi_{3}\right)$, which are functions of $t$ and $\sigma$. For applications, we have chosen $\rho_{1}^{1}=\xi_{1}, \rho_{1}^{2}=\eta_{1}$ and $\rho_{1}^{3}=\zeta_{1}$.

4. The elements of $\mathbf{D}$ appear only through the adjoint representation. Because it is a morphism of groups, it is sufficient to express $A d(\exp X)$ only for $X \in \mathcal{B}_{1}$ and to give its matrix in $\mathcal{B}_{1}$.

- If

$u=\left(\begin{array}{l}a \\ b \\ c \\ 0 \\ 0 \\ 0\end{array}\right)$

is a constant field given in $\mathcal{B}_{1}$, then:

$\operatorname{Ad}(\exp u)=\left(\begin{array}{cccccc}1 & 0 & 0 & 0 & c & -b \\ 0 & 1 & 0 & -c & 0 & a \\ 0 & 0 & 1 & b & -a & 0 \\ 0 & 0 & 0 & 1 & 0 & 0 \\ 0 & 0 & 0 & 0 & 1 & 0 \\ 0 & 0 & 0 & 0 & 0 & 1\end{array}\right)$. 
- If

$u=\xi_{1}=\left(\begin{array}{l}0 \\ 0 \\ 0 \\ 1 \\ 0 \\ 0\end{array}\right)$

then:

$A d(\exp \alpha u)$

$$
=\left(\begin{array}{cccccc}
1 & 0 & 0 & 0 & 0 & 0 \\
0 & \cos (\alpha) & -\sin (\alpha) & 0 & 0 & 0 \\
0 & \sin (\alpha) & \cos (\alpha) & 1 & 0 & 0 \\
0 & 0 & 0 & 1 & 0 & 0 \\
0 & 0 & 0 & 0 & \cos (\alpha) & -\sin (\alpha) \\
0 & 0 & 0 & 0 & \sin (\alpha) & \cos (\alpha)
\end{array}\right) .
$$

- If

$u=\eta_{1}=\left(\begin{array}{l}0 \\ 0 \\ 0 \\ 0 \\ 1 \\ 0\end{array}\right)$

then:

$A d(\exp \alpha u)$

$$
=\left(\begin{array}{cccccc}
\cos (\alpha) & 0 & \sin (\alpha) & 0 & 0 & 0 \\
0 & 1 & 0 & 0 & 0 & 0 \\
-\sin (\alpha) & 0 & \cos (\alpha) & 0 & 0 & 0 \\
0 & 0 & 0 & \cos (\alpha) & 0 & -\sin (\alpha) \\
0 & 0 & 0 & 0 & 1 & 0 \\
0 & 0 & 0 & -\sin (\alpha) & 0 & \cos (\alpha)
\end{array}\right) .
$$

- If

$u=\zeta_{1}=\left(\begin{array}{l}0 \\ 0 \\ 0 \\ 0 \\ 0 \\ 1\end{array}\right)$

then:

\section{$A d(\exp \alpha u)$}

$$
=\left(\begin{array}{cccccc}
\cos (\alpha) & -\sin (\alpha) & 0 & 0 & 0 & 0 \\
\sin (\alpha) & \cos (\alpha) & 1 & 0 & 0 & 0 \\
0 & 0 & 1 & 0 & 0 & 0 \\
0 & 0 & 0 & \cos (\alpha) & -\sin (\alpha) & 0 \\
0 & 0 & 0 & \sin (\alpha) & \cos (\alpha) & 0 \\
0 & 0 & 0 & 0 & 0 & 1
\end{array}\right) .
$$

5. For the Lie bracket, the matrix of the brackets is the following:

$$
\left(\begin{array}{cccccc}
0 & \xi_{1} & -\eta_{1} & 0 & \mathbf{k}_{1} & -\mathbf{j}_{1} \\
-\zeta_{1} & 0 & \xi_{1} & -\mathbf{k}_{1} & 0 & \mathbf{i}_{1} \\
\eta_{1} & -\xi_{1} & 0 & \mathbf{j}_{1} & -\mathbf{i}_{1} & 0 \\
0 & \mathbf{k}_{1} & -\mathbf{j}_{1} & 0 & 0 & 0 \\
-\mathbf{k}_{1} & 0 & \mathbf{i}_{1} & 0 & 0 & 0 \\
\mathbf{j}_{1} & -\mathbf{i}_{1} & 0 & 0 & 0 & 0
\end{array}\right)
$$

and the rest is deduced by bilinearity.

6. Concerning differentiations, if $v(t, \sigma)=$ $\left(v_{i}(t, \sigma)\right)_{i=1, \ldots, 6}$ is a vector of $\mathcal{D}$ given in $\mathcal{B}_{1}$, we obtain:

$\frac{\partial v(t, \sigma)}{\partial t}=\left(\frac{\partial v_{i}(t, \sigma)}{\partial t}\right)_{i=1, \ldots, 6}$,

$\frac{\partial v(t, \sigma)}{\partial \sigma}=\left(\frac{\partial v_{i}(t, \sigma)}{\partial \sigma}\right)_{i=1, \ldots, 6}+([\omega, v])_{i=1, \ldots, 6}$

where the vector $\omega$ belongs to the data of the problem and is function of $D_{r}(\sigma)$ defining the configuration of reference of the system. Here with the choice of a rectilinear configuration of reference, the matrix in $\mathcal{B}_{1}$ of the operator $v \mapsto[\omega, v]$ is given by:

$$
\left(\begin{array}{cccccc}
0 & 0 & 0 & 0 & 0 & 0 \\
0 & 0 & 0 & 0 & -1 & 0 \\
0 & 0 & 0 & 1 & 0 & 0 \\
0 & 0 & 0 & 0 & 0 & 0 \\
0 & 0 & 0 & 0 & 0 & 0 \\
0 & 0 & 0 & 0 & 0 & 0
\end{array}\right) .
$$

7. To generate a vector $v^{c}(\sigma, t)$ as function of the unknowns and their derivatives, it is necessary to differentiate relation (2). We get:

$$
\begin{aligned}
v^{c}= & A d\left(\exp \left(\psi_{1} \rho_{1}^{1}\right) \circ \exp \left(\psi_{2} \rho_{1}^{2}\right) \circ \exp \left(\psi_{3} \rho_{1}^{3}\right)\right)^{-1} \\
& \times\left(\dot{x} \mathbf{i}_{1}+\dot{y} \mathbf{j}_{1}+\dot{z} \mathbf{k}_{1}\right) \\
& +A d\left(\exp \left(\psi_{2} \rho_{1}^{2}\right) \circ \exp \left(\psi_{3} \rho_{1}^{3}\right)\right)^{-1} \dot{\psi}_{1} \rho_{1}^{1} \\
& +A d\left(\exp \left(\psi_{3} \rho_{1}^{3}\right)\right)^{-1} \dot{\psi}_{2} \rho_{1}^{2}+\dot{\psi}_{3} \rho_{1}^{3}
\end{aligned}
$$

without forgetting that for elementary motions (for example, if $X$ is a basis vector as used here), $A d(\exp (\alpha X))^{-1}=A d(\exp (-\alpha X))$, that $A d(A \circ B)=$ $A d(A) \circ A d(B)$ and that $(A \circ B)^{-1}=B^{-1} \circ A^{-1}$.

8 . To generate the vector $e^{c}(\sigma, t)$ is similar but a litthe more complicated than previously because vectors of $\mathcal{B}_{1}$ are functions of $\sigma$, too. More precisely, we have 
to calculate the derivative of $\exp (q(\sigma) X(\sigma))$ with respect to $\sigma$. Its general expression is:

$$
\begin{aligned}
& \gamma_{\exp (q(\sigma) X(\sigma))^{-1}}^{T} \frac{d(\exp (q(\sigma) X(\sigma)))}{d \sigma} \\
& \quad=\left(\frac{d q}{d \sigma} X+\sum_{m=0}^{\infty} q^{m+1} \frac{(-1)^{m}}{(m+1) !} a d^{m}(X)\left(\frac{d X}{d \sigma}\right)\right) .
\end{aligned}
$$

This sum can not always be calculated. Here, considering the rectilinear configuration of reference, we have $\frac{d X}{d \sigma}=0$ for $X=\xi, \mathbf{i}, \mathbf{j}, \mathbf{k}, \frac{d \eta}{d \sigma}=\mathbf{k}$ and $\frac{d \zeta}{d \sigma}=-\mathbf{j}$. These results let us calculate the right-hand side of (4).

As a result,

$$
\begin{aligned}
& a d^{2 p}(\eta)(\mathbf{k})=(-1)^{p} \mathbf{k}, \\
& a d^{2 p+1}(\eta)(\mathbf{k})=(-1)^{p} \mathbf{i}, \\
& a d^{2 p}(\zeta)(-\mathbf{j})=(-1)^{p}(-\mathbf{j}), \\
& a d^{2 p+1}(\zeta)(\mathbf{k})=(-1)^{p} \mathbf{i} .
\end{aligned}
$$

From these equalities, we deduce:

$$
\begin{aligned}
& \gamma_{\exp (q(\sigma) \eta(\sigma))^{-1}}^{T} \frac{d(\exp (q(\sigma) \eta(\sigma)))}{d \sigma} \\
& =\frac{d q}{d \sigma} \eta+\sin (q) \mathbf{k}+(\cos (q)-1) \mathbf{i}, \\
& \gamma_{\exp (q(\sigma) \zeta(\sigma))^{-1}}^{T} \frac{d(\exp (q(\sigma) \zeta(\sigma)))}{d \sigma} \\
& =\frac{d q}{d \sigma} \zeta-\sin (q) \mathbf{j}+(\cos (q)-1) \mathbf{i} .
\end{aligned}
$$

Equation (3) giving $v^{c}$ has to be modified in the following way:

$$
\begin{aligned}
e^{c}= & A d\left(\exp \left(\psi_{1} \rho_{1}^{1}\right) \circ \exp \left(\psi_{2} \rho_{1}^{2}\right) \circ \exp \left(\psi_{3} \rho_{1}^{3}\right)\right)^{-1} \\
& \times\left(x^{\prime} \mathbf{i}_{1}+y^{\prime} \mathbf{j}_{1}+z^{\prime} \mathbf{k}_{1}\right) \\
& +A d\left(\exp \left(\psi_{2} \rho_{1}^{2}\right) \circ \exp \left(\psi_{3} \rho_{1}^{3}\right)\right)^{-1}\left(\psi_{1}^{\prime} \rho_{1}^{1}\right) \\
& +A d\left(\exp \left(\psi_{3} \rho_{1}^{3}\right)\right)^{-1}\left(\psi_{2}{ }^{\prime} \rho_{1}^{2}+\sin \left(\psi_{2}\right) \mathbf{k}\right. \\
& \left.+\left(\cos \left(\psi_{2}\right)-1\right) \mathbf{i}\right)+\psi_{3}{ }^{\prime} \rho_{1}^{3}-\sin \left(\psi_{3}\right) \mathbf{j} \\
& +\left(\cos \left(\psi_{3}\right)-1\right) \mathbf{i} .
\end{aligned}
$$

9. The matrix $\mathbf{H}_{r}$ of the inertia operator $H_{r}$ in the basis $\mathcal{B}_{1}$ depends on the data of the problem. Let $G_{1}$ be the inertia center at the configuration of reference. Suppose the matrix of the inertia operator at $A_{1}$ in the basis $\left(\vec{i}_{1}, \vec{j}_{1}, \vec{k}_{1}\right)$ is $J_{3}$, then $\left(\operatorname{Id}_{3}\right.$ the identity matrix $3 \times 3)$ :

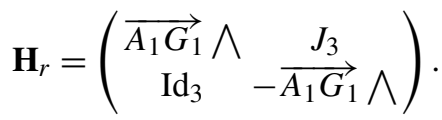

10. The coordinates $\theta_{i}$ of $\Theta^{c}$ in $\mathcal{B}_{1}$ constitute the dynamic unknowns of the system.

We give now the corresponding Maple procedures.

\section{Maple procedures}

$>\xi:=\operatorname{Vector}([0,0,0,1,0,0])$

$\eta:=\operatorname{Vector}([0,0,0,0,1,0])$

$\zeta:=\operatorname{Vector}([0,0,0,0,0,1])$

$i c:=\operatorname{Vector}([1,0,0,0,0,0])$;

$j c:=\operatorname{Vector}([0,1,0,0,0,0])$;

$k c:=\operatorname{Vector}([0,0,1,0,0,0])$;

$\sharp$ vectors setting up the basis $\mathcal{B}_{1}$

$>c l:=\operatorname{proc}(X, Y) \sharp$ procedure giving in $\mathcal{B}_{1}$ the Lie bracket of two vectors $X, Y$ given in $\mathcal{B}_{1}$ too

$>\operatorname{eval}((X[1] * Y[5]-X[5] * Y[1]) * k c+(X[1] *$ $Y[6]-X[6] * Y[1]) *(-j c)+(X[2] * Y[4]-X[4] *$ $Y[2]) *(-k c)+(X[2] * Y[6]-X[6] * Y[2]) * i c+$ $(X[3] * Y[4]-X[4] * Y[3]) * j c+(X[3] * Y[5]-$ $X[5] * Y[3]) *(-i c)+(X[4] * Y[5]-X[5] * Y[4]) *$ $\zeta+(X[4] * Y[6]-X[6] * Y[4]) *(-\eta)+(X[5] *$ $Y[6]-X[6] * Y[5]) * \xi)$;

$>$ end proc;

$>$ Adel $:=\operatorname{proc}(a, X) \sharp$ procedure providing in $\mathcal{B}_{1}$ matrices of adjoint mappings $\operatorname{Ad}(\exp (a X))$ for vectors of bais $\mathcal{B}_{1}$

$>$ if eval $(X)=\operatorname{eval}(i c)$ then $\operatorname{Matrix}([[1,0,0,0,0,0]$, $[0,1,0,0,0, a],[0,0,1,0,-a, 0],[0,0,0,1,0,0]$, $[0,0,0,0,1,0],[0,0,0,0,0,1]])$; $>$ elif $X=j c$ then Matrix ([[1, 0, 0, 0, 0, $-a]$, $[0,1,0,0,0,0],[0,0,1, a, 0,0],[0,0,0,1,0,0]$, $[0,0,0,0,1,0],[0,0,0,0,0,1]])$; $>$ elif $X=k c$ then Matrix ([[1, 0, 0, 0, $a, 0]$, $[0,1,0,-a, 0,0],[0,0,1,0,0,0],[0,0,0,1,0,0]$, $[0,0,0,0,1,0],[0,0,0,0,0,1]])$; $>$ elif $X=\xi$ then Matrix ([[1, 0, 0, 0, 0, 0], $[0, \cos (a),-\sin (a), 0,0,0]$, $[0, \sin (a), \cos (a), 0,0,0]$, $[0,0,0,1,0,0],[0,0,0,0, \cos (a),-\sin (a)]$, $[0,0,0,0, \sin (a), \cos (a)]])$; 
$>$ elif $X=\eta$ then Matrix $([[\cos (a), 0, \sin (a), 0,0,0]$,

$[0,1,0,0,0,0],[-\sin (a), 0, \cos (a), 0,0,0]$,

$[0,0,0, \cos (a), 0, \sin (a)],[0,0,0,0,1,0]$,

$[0,0,0,-\sin (a), 0, \cos (a)]])$;

$>$ elif $X=\zeta$ then

$\operatorname{Matrix}([[\cos (a),-\sin (a), 0,0,0,0]$,

$[\sin (a), \cos (a), 0,0,0,0],[0,0,1,0,0,0]$,

$[0,0,0,0, \cos (a),-\sin (a)]$,

$[0,0,0,0, \sin (a), \cos (a)],[0,0,0,0,0,1]])$;

$>$ end if;

$>$ end proc;

$>H r:=\operatorname{proc}(B, \lambda, \mu, v) \sharp$ procedure giving the matrix of $H_{r}$ in $\mathcal{B}_{1}$ where $B$ is the matrix of inertia at the origin in $\mathcal{B}_{1}$ and $\lambda, \mu, v$ the coordinates of the center of inertia in $\mathcal{R}_{1}$

$>\operatorname{Matrix}([[0, v,-\mu, B[1,1], B[1,2], B[1,3]]$,

$[-v, 0, \lambda, B[2,1], B[2,2], B[2,3]]$,

$[\mu,-\lambda, 0, B[3,1], B[3,2], B[3,3]]$,

$[1,0,0,0,-v, \mu],[0,1,0, v, 0,-\lambda]$,

$[0,0,1,-\mu, \lambda, 0]])$

$>$ end proc;

$>$ Diffe $:=\operatorname{proc}(X, v a r) \sharp$ procedure giving the derivation with respect to the variable var of a vector $X$ given in $\mathcal{B}_{1}$

$>$ local $M$;

$>$ if $v a r=s$ then $M:=$ Matrix $([[0,0,0,0,0,0]$,

$[0,0,0,0,0,-1],[0,0,0,0,1,0],[0,0,0,0,0,0]$,

$[0,0,0,0,0,0],[0,0,0,0,0,0]])$

> else $M=\operatorname{Matrix}([[0,0,0,0,0,0],[0,0,0,0,0,0]$,

$[0,0,0,0,0,0],[0,0,0,0,0,0]$,

$[0,0,0,0,0,0],[0,0,0,0,0,0]])$ end if;

$>\operatorname{eval}(\operatorname{Vector}([\operatorname{diff}(X[1]$, var $), \operatorname{diff}(X[2]$, var $)$,

$\operatorname{diff}(X[3], v a r), \operatorname{diff}(X[4], v a r)$,

$\operatorname{diff}(X[5], v a r), \operatorname{diff}(X[6], v a r)])+$

Matrix VectorMultiply $(M, X))$;

$>$ end proc;

$>V c:=\operatorname{proc}(a, b, c, d, e, f) \sharp$ procedure providing velocity $v^{c}$ in $\mathcal{B}_{1}$

> local $A, V 1, V 2, V 3$;

$>A:=\operatorname{Adel}(-f, \zeta)$

$>V 1:=$ Matrix VectorMultiply $(A, \eta)$

$>A:=A \cdot A d e l(-e$, eta $)$;

$>V 2:=$ Matrix VectorMultiply $(A, \xi)$;

$>A:=A \cdot \operatorname{Adel}(-d, x i)$;

$>V 3:=\operatorname{diff}(a, t) * i c+\operatorname{diff}(b, t) * j c+\operatorname{diff}(c, t) * k c ;$

$>$ Matrix VectorMultiply $(A, V 3)+\operatorname{diff}(d, t) * V 2+$ $\operatorname{diff}(e, t) * V 1+\operatorname{diff}(f, t) * \zeta$

$>$ end proc;

$>E c:=\operatorname{proc}(a, b, c, d, e, f) \sharp$ procedure providing strain $e^{c}$ in $\mathcal{B}_{1}$

> local $A, U 1, U 2, U 3, U 4$;

$>A:=\operatorname{Adel}(-f, \zeta)$

$>U 1:=$ Matrix VectorMultiply $(A, \eta)$;

$>U 4:=$ MatrixVectorMultiply $(A, \sin (e) * k c+$ $(\cos (e)-1) * i c)$;

$>A:=A \cdot \operatorname{Adel}(-e, \eta)$;

$>U 2:=\operatorname{Matrix} V e c t o r M u l t i p l y(A, \xi)$;

$>A:=$ A.Adel $(-d, \xi)$;

$>U 3:=\operatorname{diff}(a, s) * i c+\operatorname{diff}(b, s) * j c+\operatorname{diff}(c, s) *$

$k c$;

$>$ Matrix VectorMultiply $(A, U 3)+\operatorname{diff}(d, s) * U 2+$ $\operatorname{diff}(e, s) * U 1+U 4+\operatorname{diff}(f, s) * \zeta-\sin (f) * k c+$ $(\cos (f)-1) * i c$;

$>$ end proc;

$>E Q:=\operatorname{proc}(r, I C, x G, y G, z G, X, T i, T e) \sharp$ procedure providing equations where $r$ is linear density of mass, IC central matrix of inertia of section in $\mathcal{R}_{1}, x G, y G, z G$ coordinates of $G$ in $\mathcal{R}_{1}, X$ vector kinematic unknowns, $T i$ Lagrangian vector of internal strengths in $\mathcal{B}_{1}, T e$ Lagrangian vector of external strengths in $\mathcal{B}_{1}$

$>$ local $T$, Theta, ec, vc, ac, $M, H, K, D, i, e q$;

$>e c:=E c(X[1], X[2], X[3], X[4], X[5], X[6])$;

$>v c:=V c(X[1], X[2], X[3], X[4], X[5], X[6]) ;$

$>a c:=\operatorname{Diffe}(v c, t)$

$>T:=T e$;

$>$ Theta $:=T i$;

$>M:=\operatorname{eval}(H r(I C, x G, y G, z G))$;

$>H:=\operatorname{eval}($ MatrixVectorMultiply $(M, v c))$;

$>K:=\operatorname{eval}($ Matrix VectorMultiply $(M, v c)+$

$\operatorname{cl}(H, v c))$

$>D:=\operatorname{eval}($ Diffe $($ Theta,$s)+\operatorname{cl}($ Theta,$e c))$;

$>e q:=-r * K+r * T+D$;

$>$ end proc;

\section{An example}

To conclude and to illustrate the previous section, one of the six equations is given (see Fig. 1).

$>\operatorname{Incin}:=(s, t)->\operatorname{Vector}([u(s, t), v[1](s, t)$, $v[2](s, t), \theta(s, t), \phi[1](s, t), \phi[2](s, t)]) ; \sharp$ vector of kinematic unknowns 


$$
\begin{aligned}
& -\rho\left(I\left(\left(\frac{\partial}{\partial t} \theta(s, t)\right) \sin \left(\varphi_{2}(s, t)\right) \sin \left(\varphi_{1}(s, t)\right)+\left(\frac{\partial}{\partial t} \varphi_{1}(s, t)\right) \cos \left(\varphi_{2}(s, t)\right)\right)+J\left(( \frac { \partial } { \partial t } \theta ( s , t ) ) \operatorname { c o s } ( \varphi _ { 2 } ( s , t ) ) \operatorname { s i n } \left(\varphi_{1}(\right.\right.\right. \\
& \left.s, t)) \cdot\left(\frac{\partial}{\partial t} \varphi_{1}(s, t)\right) \sin \left(\varphi_{2}(s, t)\right)\right)\left(\left(\frac{\partial}{\partial t} \theta(s, t)\right) \sin \left(\varphi_{1}(s, t)\right)+\left(\frac{\partial}{\partial t} \varphi_{2}(s, t)\right)\right) \cdot K\left(( \frac { \partial } { \partial t } \theta ( s , t ) ) \operatorname { s i n } \left(\varphi_{1}(\right.\right. \\
& \left.\left.s, t))+\left(\frac{\partial}{\partial t} \varphi_{2}(s, t)\right)\right)\left(\left(\frac{\partial}{\partial t} \theta(s, t)\right) \cos \left(\varphi_{2}(s, t)\right) \sin \left(\varphi_{1}(s, t)\right) \cdot\left(\frac{\partial}{\partial t} \varphi_{1}(s, t)\right) \sin \left(\varphi_{2}(s, t)\right)\right)\right)+\rho v(s, t) \\
& +\left(\frac{\partial}{\partial s} N(s, t)\right)+T_{1}(s, t)\left(\left(\frac{\partial}{\partial s} \theta(s, t)\right) \sin \left(\varphi_{1}(s, t)\right)+\left(\frac{\partial}{\partial s} \varphi_{2}(s, t)\right)\right)-M_{2}(s, t)( \\
& -\sin \left(\varphi_{2}(s, t)\right) \cos \left(\varphi_{1}(s, t)\right)\left(\frac{\partial}{\partial s} u(s, t)\right)
\end{aligned}
$$$$
+\left(\cos \left(\varphi_{2}(s, t)\right) \cos (\theta(s, t))-\sin \left(\varphi_{2}(s, t)\right) \sin \left(\varphi_{1}(s, t)\right) \sin (\theta(s, t))\right)\left(\frac{\partial}{\partial s} v_{1}(s, t)\right)
$$$$
+\left(\cos \left(\varphi_{2}(s, t)\right) \sin (\theta(s, t))+\sin \left(\varphi_{2}(s, t)\right) \sin \left(\varphi_{1}(s, t)\right) \cos (\theta(s, t))\right)\left(\frac{\partial}{\partial s} v_{2}(s, t)\right) \cdot \sin \left(\varphi_{2}(s, t)\right)\left(\cos \left(\varphi_{1}(s, t)\right)-1\right)
$$$$
\int-T_{2}(s, t)\left(\left(\frac{\partial}{\partial s} \theta(s, t)\right) \cos \left(\varphi_{2}(s, t)\right) \sin \left(\varphi_{1}(s, t)\right) \cdot\left(\frac{\partial}{\partial s} \varphi_{1}(s, t)\right) \sin \left(\varphi_{2}(s, t)\right)\right)+M_{1}(s, t)\left(\sin \left(\varphi_{1}(s, t)\right)(\right.
$$$$
\left.\frac{\partial}{\partial s} u(s, t)\right)-\cos \left(\varphi_{1}(s, t)\right) \sin (\theta(s, t))\left(\frac{\partial}{\partial s} v_{1}(s, t)\right)+\cos \left(\varphi_{1}(s, t)\right) \cos (\theta(s, t))\left(\frac{\partial}{\partial s} v_{2}(s, t)\right)+\sin \left(\varphi_{1}(s, t)\right)
$$$$
-\sin \left(\varphi_{2}(s, t)\right)
$$

Fig. 1 Equation (1)

$>\operatorname{Indyn}:=(s, t)->\operatorname{Vector}([N(s, t), T[1](s, t)$, $T[2](s, t), C(s, t), M[1](s, t), M[2](s, t)]) ; \sharp \quad$ vector of internal unknowns

$>$ Efext $:=(s, t)->\operatorname{Vector}([v(s, t), \tau[1](s, t)$, $\tau[2](s, t), \Gamma(s, t), C[1](s, t), C[2](s, t)]) ; \sharp$ vector of external strengths

In := Matrix $([[I, 0,0],[0, J, 0],[0,0, K]]) ; \sharp$ matrix of Inertia operator

equations $\quad:=\quad E Q($ rho, In, 0, 0, 0, Incin $(s, t)$, $\operatorname{Indyn}(s, t), \operatorname{Efext}(s, t)) ; \sharp$ equations of curvilinear system. Center of inertia is the origin of frame.

\section{Conclusion}

From previous papers that contain the intrinsic formulation of the dynamics of curvilinear systems by using calculations in Lie groups, we have developed here the steps for the automatic obtainment of explicit scalar equations. First, the main points are specified and necessary additional formulae and calculations in the Lie group of Euclidean displacements are made. Second, the corresponding Maple procedures are given. The complexity of these derived equations lead us to think that a "handmade" method would be inconceivable. It 
should also be noted here that such an approach leads to an easy numerical treatment (for example directly with Maple), under reserve to add a constitutive law as previously remarked.

\section{References}

1. Chevallier, D.P.: Lie algebras, modules, dual quaternions and algebraic methods in kinematics. Mech. Mach. Theory 26(6), 613-627 (1991)
2. Chevallier, D.P.: Groupes de Lie et Mécanique des systèmes de corps rigides. In: Séminaire Modélisation Mathématique, Kassel, 1-4 Octobre 1984, pp. 231-270. McGraw-Hill, New York (1986)

3. Lerbet, J.: Generation of general equations of curvilinear systems. A coordinate-free approach by using Lie group theory. Mech. Res. Commun. 30, 505-512 (2003)

4. Lerbet, J.: Non-linear dynamics of curvilinear systems. A new coordinateless approach. J. Appl. Math. Mech. 69, 917-924 (2005) 